\title{
EFECTO DE LA CANTIDAD DE REPETICIONES MENTALES DIRIGIDAS DE LA TAREA SOBRE EL RENDIMIENTO DE UNA RUTINA OBLIGATORIA DE PISO EN NIÑAS QUE PRACTICAN GIMNASIA
}

\author{
Laura Sobalvarro Chavarría y Gerardo Araya Vargas \\ Escuela de Educación Física y Deportes \\ Universidad de Costa Rica, San José Costa Rica \\ E-mail: laurasobalvarro@costarricense.com
}

\begin{abstract}
Resumen
Sobalvarro, L., y Araya, G. (2001). Efecto de la cantidad de repeticiones mentales dirigidas de la tarea sobre el rendimiento de una rutina obligatoria de piso en niñas que practican gimnasia. Revista en Ciencias del Ejercicio y la Salud, 1(2), 1924. El propósito de esta investigación fue comparar dos cargas diferentes de práctica mental, una en que se realizaba dos repeticiones mentales de una rutina de piso y otra en la cual se hacía cinco repeticiones, para observar su efecto sobre el rendimiento en la ejecución de una rutina de piso en gimnasia. Participaron 18 niñas con edades entre cinco y doce años quienes practicaban gimnasia regularmente tres veces por semana desde al menos seis meses antes del estudio y que entrenaban para alcanzar los niveles III y IV de la USAG. Estas niñas se dividieron en dos grupos experimentales y un grupo control. El tratamiento de los grupos experimentales consistió en ver el video de la rutina, hacer práctica mental según el protocolo que les correspondía, practicar la rutina y ser evaluadas. Esto en cada sesión, para un total de 10 sesiones, que se desarrollaron tres veces por semana. El grupo control en cambio no realizó práctica mental ni física de la rutina solo veía el video, hacía una vez la rutina y era evaluado, pero esto fue solo en tres ocasiones (sesiones 1, 6 y 10); el resto del tiempo continuó con sus entrenamientos habituales. Dentro de los instrumentos de medición utilizados están el protocolo técnico de la rutina de piso y el protocolo de entrenamiento mental. Los resultados del ANOVA de dos vías (Grupos experimentales por 10 mediciones) demostraron que al considerar las 10 mediciones se observa que el grupo experimental dos obtuvo un rendimiento significativamente mayor que el grupo experimental uno, $(F: 28.10, p<0.05)$ y en ambos grupos los mejores niveles de rendimiento se observan de la quinta a la sétima sesión, (F:23.86, $p<0.05)$. Los resultados del ANOVA de dos vías (Grupo control y experimentales en tres mediciones repetidas) demostraron que a lo largo de las mediciones, el grupo control no tuvo cambios significativos a diferencia de los experimentales, que mostraron un mejor rendimiento en la sexta y décima medición (F:20.09, $p<0.05)$. Se concluye que la práctica mental es más efectiva si se realiza mayor cantidad de repeticiones de la tarea antes de practicar físicamente. Además se recomienda combinar la práctica mental con la práctica física en niñas gimnastas para obtener un mejor rendimiento en su práctica deportiva. PALABRAS CLAVES: Entrenamiento mental, gimnasia, niñas, rutinas de piso, imaginería, práctica mental.
\end{abstract}

\section{INTRODUCCIÓN}

Todo el mundo tiene la habilidad para crear imágenes mentales pero no todos se dan cuenta del potencial que tiene su aplicación sistemática, para optimizar procesos de aprendizaje motor, estimular capacidades cognitivas en asociación con el ejercicio físico o provocar mejoras en los mecanismos de regulación emocional.

White y Hardy (1998) señalan que el uso de imágenes mentales reproduce la experiencia real, donde se puede ver o sentir movimientos o experimentar sabores, olores o sonidos sin estar viviendo realmente esas cosas. Esto se diferencia de un sueño en cuanto a que la persona está consciente cuando forma una imagen mental.

Betts (1909) realizó uno de los primeros estudios en esta área. Analizó varias tareas donde se utilizaban imágenes mentales de forma espontánea. Entre las tareas tenemos la asociación simple, pensamiento lógico, multiplicación mental y juicios de discriminación. Él encontró que las imágenes mentales se usaban frecuentemente en esas tareas, pero que eran más útiles en unas que en otras.

A finales del siglo XIX algunos investigadores empezaron a preocuparse por analizar 
la actividad muscular durante el desarrollo de procesos de operaciones mentales, uno de estos pioneros fue Jastrow (1892), quien estudió la actividad muscular en movimientos involuntarios.

En la década de los treinta del siglo XX se iniciaron líneas de investigación (Sacket,1934; Jacobson,1932; Perry, 1939) que se preocupaban por examinar el uso de la práctica mental, muchas de ellas enfocadas hasta la década de los ochenta, en los efectos de la práctica mental, sobre el aprendizaje y rendimiento de destrezas motrices. Murphy y Jowdy, (1992) señalan que toda esta gran producción de investigación se fundamentó en trabajos de laboratorio principalmente.

Se han propuesto dos grandes teorías para tratar de explicar los efectos de la práctica mental. Una de ellas es la Teoría Psiconeuromuscular fundamentada en gran cantidad de estudios (Jastrow, 1892; Washburn,1916; Harris y Robinson,1986) que han probado que cuando una persona imagina que está moviendo alguna parte de su cuerpo, los músculos específicos asociados con el movimiento que está siendo imaginado, tienen variaciones en su actividad mioeléctrica. Esto significa que existe relación entre las imágenes mentales y la inervación de los músculos. Sin embargo este enfoque no permite explicar todos los efectos de la práctica mental.

La Teoría del Aprendizaje Simbólico establece que la práctica mental beneficia el rendimiento motriz debido a que los sujetos se preparan cognitivamente para la actividad y planean la tarea que van a realizar (Horn, 1992). Murphy y Jowdy (1992) señalan que existe evidencia de que la práctica mental es mucho más efectiva para tareas que tienen un elevado componente cognitivo, como sería el caso de una rutina de gimnasia de piso, por ejemplo.

En este sentido, Feltz y Landers, (1983) realizaron un metaanálisis con 60 estudios de los cuales se obtuvo 146 tamaños de efecto. Encontraron un tamaño de efecto promedio de 0,48 lo cual sugiere que la práctica mental es mejor que no practicar del todo y además los tamaños de efecto más grandes, se encontraron en tareas cognitivas más que en tareas motoras $\mathrm{o}$ de resistencia.
Murphy y Jowdy, (1992) recalcan que la evidencia científica actual muestra que la práctica mental es mejor que no hacerla del todo y que una combinación de la práctica mental y física es mejor que hacer solo práctica física.

Paivio (1985) propuso que el uso de imágenes mentales influencia el rendimiento y el aprendizaje a través no solo de actividades cognitivas sino también motivacionales, siendo útil en este caso para mejorar la motivación hacia destrezas específicas, incrementar la motivación en general mediante la creación de un estado anímico óptimo para la actividad. Este aspecto es clave, sobre todo al trabajar con niños menores a $10 \mathrm{u} 11$ años.

Específicamente en niños, muy pocos estudios sobre práctica mental se han realizado. Diversos autores (Weinberg y Gould, 1995) han reportado que los niños al iniciar la práctica en el deporte lo hacen con un fin de recreación más que de competición, por tanto, muchos podrían pensar que la aplicación de técnicas de preparación mental, en sujetos infantiles, podría ser poco fructífera.

Sin embargo, estudios previos realizados con niñas gimnastas de Centroamérica y el Caribe durante competencias de nivel Panamericano y Centroamericano han demostrado que a la hora de competir las gimnastas presentan niveles altos de tensión, depresión y hostilidad, además de reportar que no se tenía ánimo para competir; también se ha reportado que el tipo de competencia influye en el estado anímico que las gimnastas presentan, pues en competencias de mayor nivel el rendimiento tiende a ser más bajo que en competencias de menor nivel (Sobalvarro y Salazar,1998 y 1999). Por lo tanto se podría esperar que la aplicación de un entrenamiento mental en niñas que practican gimnasia podría provocar mejoras en su rendimiento en general.

En este sentido, Murphy y Jowdy (1992) señalan que, se requieren más estudios que permitan conocer qué características debe tener la práctica mental, para ser más efectiva, conocimiento que permitiría desarrollar programas de entrenamiento mental más sólidos. Una de estas características es el volumen y la frecuencia con la que se pueda realizar la práctica mental. Específicamente sobre el volumen, esta variable no ha sido tomada en cuenta en estudios previos. 
El volumen puede considerarse como la duración del ejercicio de práctica mental o como la cantidad de repeticiones de una misma secuencia mental, el cual es el aspecto bajo el que es enfocado el volumen en este estudio.

También, cabe anotar la poca cantidad de estudios realizados con niños menores de 12 años (Feltz y Landers, 1983; Weinberg y Gould, 1995). Debido a lo anterior, el propósito de esta investigación es comparar dos cargas diferentes de práctica mental, uno en que se realiza dos repeticiones mentales de una rutina de piso y otro en el cual se hace cinco repeticiones, para observar su efecto sobre el rendimiento en la ejecución de la rutina.

\section{METODOLOGÍA}

\section{Sujetos}

Participaron 18 niñas con edades entre cinco $\mathrm{y}$ doce años quienes practicaban gimnasia regularmente tres veces por semana desde al menos seis meses antes del estudio y que entrenaban para alcanzar los niveles III y IV de la USA Gymnastics (USAG).

\section{Instrumentos}

Para realizar esta investigación se requirió de un protocolo técnico el cual fue el video de la rutina de los niveles III y IV de la USAG para la referencia de un modelo y un protocolo de práctica mental el cual fue escrito (ver cuadro 1) y grabado con indicaciones para la misma y descripción de la rutina. La grabación era reproducida por medio de una grabadora. Además se solicitó la ayuda de una Juez Internacional de Gimnasia para evaluar el rendimiento de las niñas en la rutina.

\section{Procedimiento}

Las niñas que participaron fueron asignadas al azar a dos grupos experimentales. Mientras que el grupo control ya estaba previamente formado. Las niñas del grupo experimental 1 (2PM) vieron el video de la rutina una vez, hicieron dos repeticiones mentales de la rutina de piso (según protocolo que se describe en el cuadro 1) en forma continua y sin pausas y luego realizaron práctica física de la rutina una vez para, posteriormente, pasar a ser evaluadas y continuar con su entrenamiento regular, que era igual para los otros grupos.

Las niñas del grupo experimental 2 (5PM) vieron el video de la rutina una vez, hicieron cinco repeticiones mentales de la rutina de piso en forma continua, luego realizaron una práctica física de la rutina y pasaron a ser evaluadas. Posteriormente las niñas continuaron con su entrenamiento normal.

A los dos grupos experimentales se les evaluó su rendimiento, como se indicó en los dos párrafos anteriores, en las diez sesiones que abarcó el estudio, las cuales tuvieron una frecuencia de 3 veces por semana. El rendimiento se evaluó siguiendo las pautas generales de evaluación en Gimnasia Artística Femenina. El protocolo que se siguió para las mediciones fue el siguiente: Se determinaba primero la nota de partida, la cual se establece con base en los elementos que se incluyen en la rutina obligatoria del nivel; si la niña llega a realizar la rutina completa con todos los elementos que esta incluye, su nota de partida será de 10 pero si la niña omite uno o varios elementos su nota de partida será más baja porque cada elemento tiene un valor el cual se resta si no lo ejecuta, así su nota puede variar de 0 a 10 . Una vez determinada la nota de partida se establecía la nota final, la cual se obtiene restándole a la nota de partida la suma total de las deducciones por ejecución y presentación artística, que la niña obtuvo en su rutina. Por ejemplo: Cuando la ejecutante presentaba su rutina ante la juez, esta la evaluaba, como ya se ha descrito; cuando la ejecutante finalizaba, la juez procedía a calcular la nota de partida y luego la nota final, según lo que se ha descrito anteriormente.

Las niñas del grupo control, a diferencia de los grupos experimentales, no realizaron práctica mental sino que pasaban de ver el video de la rutina a practicar la rutina una vez, y a ser evaluadas, esto en las sesiones que se les realizó las mediciones a saber sesiones 1,6 y 10 . 


\title{
Cuadro 1
}

\section{Protocolo de Práctica Mental de la Rutina de Piso de nivel IV USAG}

\begin{abstract}
Colóquese cómodamente, sentada, recostada, donde no sienta tensión, cierre los ojos y empiece a jugar con imágenes en la mente, tome aire por la nariz y bótelo por la boca suavemente contando $123 .$. , tome aire y ahora exhale...va a realizarlo otra vez pero con la respiración más profunda, tome aire 123 ahora exhale.... Se siente tibia, su piel está tibia, su respiración es muy relajada. Ahora puede ver en su mente un gimnasio, que tiene piso de madera, usted ahora está caminando en el gimnasio y siente muy bien como da cada paso, el piso está frío, usted lo siente mientras camina, ahora va a empezar a trotar y el piso se vuelve tibio ya no está frío, está tibio, ahora se va a detener y va a tomar la posición de saludo inicial y va a hacer su rutina: Realice una elevación de pierna y haga parada de manos sosteniendo 2 segundos con postura baje a rollo con brazos rectos y rodillas juntas, termine el movimiento y mantenga la cabeza arriba, ahora haga un fuerte take-off y realice una rondada empujando fuerte de brazos y con buen corvet ahora continúe el flic flac siempre hacia atrás con brazos rectos y un rebote bien alto, ahora prepárese para iniciar el rollo atrás a parada de manos con el pecho adentro y brazos rectos y dure 2 segundos levante su barbilla y mantenga elegancia en el movimiento, ahora eleve la pierna a la cadera y de un paso grande al frente para hacer parada de manos puente, hágalo siempre dura, mantenga los brazos rectos y deje los hombros atrás, manténgase viendo el piso siempre y los brazos suben con la cabeza. Termine bien el movimiento y prepárese para hacer los saltos, ahora haga paso salto split paso paso salto split, no olvide elevar la pierna y empujar con la cadera, además de mantener gran amplitud en el salto apenas cae del segundo salto prepárese para hacer giro de $360^{\circ}$ en un pie, hágalo y mantenga la posición de passe hasta que su pie pase al frente y no baje el talón antes de terminar el giro, termine el movimiento y mantenga buena presentación artística. Ahora con mucha elegancia y femineidad realice onda atrás y chasse con cambio de pies, ahora que terminó, bien elegante, haga pose, mantenga el tronco recto, pie en punta y flexionado y los dedos en la posición correcta, ahora haga salto extendido con medio giro salto extendido con medio giro y al ritmo de la música termine con onda hacia delante y espere dos segundos. -(2) y (5) repeticiones de esta rutina, según corresponda-. En estos momentos se siente muy contenta de lo realizado hasta ahora se siente satisfecha y tiene esa sensación de alegría que es como un calor que va por dentro de su cuerpo, ahora tome aire otra vez, fuertemente 123 , se siente fuerte y llena de energía y ahora abra los ojos lentamente.
\end{abstract}

\section{Análisis estadístico}

Para realizar el análisis estadístico de esta investigación se utilizaron diferentes medidas estadísticas: Promedio y desviación estándar de las variables medidas; análisis de varianza (ANOVA) de dos vías de medidas repetidas (2X10), grupos experimentales por mediciones en cada sesión para determinar la diferencia en el rendimiento de cada medición entre ambos grupos experimentales; ANOVA de dos vías de medidas repetidas (3X3), grupos por mediciones para comparar ambos grupos experimentales y el control en cuanto a su rendimiento en las mediciones realizadas en la primera, sexta y décima sesiones. También se aplicó análisis Post- Hoc de Tukey cuando fue necesario.

\section{RESULTADOS}

La tabla 2 presenta la estadística descriptiva (promedios y desviaciones estándar) cada medición para cada uno de los grupos.

El ANOVA $3 \times 3$ (grupos $\mathrm{x}$ mediciones) indicó que el grupo control no tuvo cambios significativos en las mediciones realizadas en las sesiones uno, seis y diez ( $p>0.05)$; mientras que los grupos experimentales sí cambiaron significativamente $(p<0.05)$ en tales mediciones (ver gráfico 1).

El ANOVA 2 x 10 (grupos $\mathrm{x}$ mediciones) indicó que durante las 10 mediciones realizadas el rendimiento fue significativamente mayor de la quinta a la sétima sesión $(\mathrm{p}<0.05)$. Y esto ocurrió tanto en el grupo que realizó 2 repeticiones de práctica mental como en el que realizó 5 repeticiones de práctica mental (ver gráfico 2).

\section{DISCUSIÓN}

Los resultados de esta investigación demuestran que, la aplicación de entrenamiento mental, en niñas, es efectiva para adquirir destrezas y mejorar el rendimiento en estas mismas, en tareas cognitivas, como una rutina de piso en gimnasia. Estos resultados están en la línea de lo señalado por el metanálisis de Feltz y Landers (1983). No obstante, una de las predicciones del metanálisis, con respecto al efecto de la práctica mental en sujetos novatos, como los de este estudio, se contradice con los resultados obtenidos. Feltz y Landers (1983), encontraron tamaños de efecto mayores en sujetos experimentados (o sea, que estaban en una etapa 
tardía del aprendizaje) en comparación con los de sujetos novatos (o sea, en etapas tempranas de aprendizaje), por lo que cabría esperar poco o ningún efecto de la práctica mental, en estos últimos, aunque la diferencia entre los tamaños de efecto respectivos no fue significativa.

Tabla 2.

Estadística Descriptiva, Promedios y Desviación Estándar de los puntajes de la rutina de cada grupo, en cada medición

\begin{tabular}{|c|c|c|c|c|c|c|c|c|c|c|}
\hline Grupos & M 1 & M 2 & M 3 & M 4 & M 5 & M 6 & M 7 & M 8 & M 9 & M 10 \\
\hline $2 \mathrm{PM}$ & $\begin{array}{l}\times 3.07 \\
\pm 0.72\end{array}$ & $\begin{array}{l}\times 3.30 \\
\pm 0.70\end{array}$ & $\begin{array}{l}x 3.83 \\
\pm 0.69\end{array}$ & $\begin{array}{l}\times 3.95 \\
+0.76\end{array}$ & $\begin{array}{l}\times 4.06 \\
+0.84\end{array}$ & $\begin{array}{l}\times 4.50 \\
+1.38\end{array}$ & $\begin{array}{l}\times 4.70 \\
+1.20\end{array}$ & $\begin{array}{l}\times 4.95 \\
+1.02\end{array}$ & $\begin{array}{l}\times 5.19 \\
\pm 0.88\end{array}$ & $\begin{array}{l}\times 5.19 \\
\pm 0.96\end{array}$ \\
\hline $5 \mathrm{PM}$ & $\begin{array}{r}\times 5.48 \\
+0.49\end{array}$ & $\begin{array}{l}\times 5.61 \\
+0.49\end{array}$ & $\begin{array}{l}x \quad 5.77 \\
+0.43\end{array}$ & $\begin{array}{l}x 5.92 \\
+0.47\end{array}$ & $\begin{array}{r}x \quad 6.44 \\
+0.59\end{array}$ & $\begin{array}{l}x \quad 6.81 \\
+0.51\end{array}$ & $\begin{array}{l}\times 6.94 \\
+0.38\end{array}$ & $\begin{array}{r}x 7.63 \\
+0.69\end{array}$ & $\begin{array}{l}x 7.54 \\
+0.55\end{array}$ & $\begin{array}{l}\times 7.60 \\
+0.56\end{array}$ \\
\hline Control & $\begin{array}{l}\times 2.30 \\
\pm 1.48\end{array}$ & & & & & $\begin{array}{l}x 2.30 \\
\pm 0.34\end{array}$ & & & & $\begin{array}{l}\times 2.40 \\
\pm 0.37\end{array}$ \\
\hline
\end{tabular}

Nota: $\mathrm{M}$ indica la medición efectuada ( una en cada sesión para los grupos experimentales y tres para el grupo control, las cuales coincidieron con las sesiones uno, seis y diez) PM es igual a práctica mental.

Sin embargo, las niñas en ambos grupos experimentales tuvieron una mejoría significativa en su rendimiento $\mathrm{y}$, además, los cambios en el rendimiento fueron rápidos, pues ya para la quinta sesión, a mediados de la segunda semana de tratamiento, se observaron diferencias signicativas, entre las mediciones del rendimiento (ver gráfico 2).

Otro aspecto importante de destacar es, que los resultados de este estudio respaldan las predicciones del metanálisis, sobre el impacto de la combinación de práctica mental y física, en la adquisición de una destreza y en el mejoramiento de su desempeño, pese a la evidencia adversa presentada por Hird, Landers, Thomas y Horan (1991) y Durand, May y Haslam (1997).

La práctica mental permite retener mejor una tarea que se está aprendiendo, según el estudio de Meacci y Price (1985), quienes encontraron que, cuando se aplica una estrategia de práctica mental, durante la fase de aprendizaje de una tarea, la retención de esta es mejor que en un grupo control, de no práctica. Al haber mayor retención de lo aprendido, e ir sumando nuevas prácticas combinadas, mentales y físicas, se esperaría una mejora progresiva y mayor, que en la condición de control, lo cual fue evidenciado por los resultados del presente estudio.

Gráfico 1

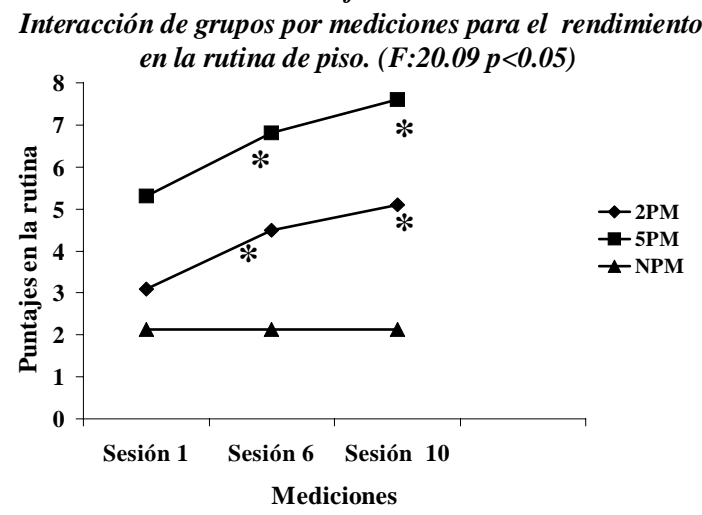

Una variable que no se ha tomado en cuenta antes, es el volumen de la práctica mental, entendido en este estudio como la cantidad de repeticiones mentales de la rutina. Este factor es clave para planificar, junto al trabajo físico y técnico táctico, todo un proceso de preparación mental.

Las niñas que realizaron mayor cantidad de repeticiones mentales obtuvieron mejores resultados que las niñas que hicieron menos repeticiones o las que no hicieron práctica mental (ver gráfico 1). Ante 
estos resultados se evidencia que las niñas que hicieron más repeticiones mentales de la rutina de piso obtuvieron mejores resultados. Sin embargo, independientemente de la cantidad de repeticiones mentales, los dos grupos que hacían práctica mental y física, tuvieron un comportamiento similar en cuanto a la aparición de las diferencias significativas, en las mediciones de rendimiento en la rutina (ver gráfico 2).

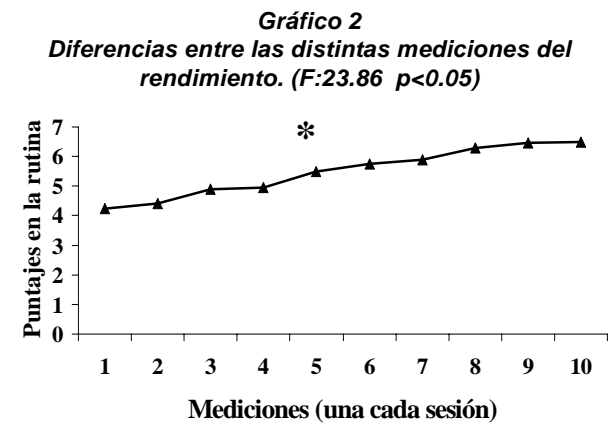

Sobalvarro y Salazar (1998, 1999) sugirieron ponerle atención a las gimnastas de Costa Rica en cuanto a características psicológicas que presentaron precompetencia, indicando la aplicación de técnicas de preparación mental, para mejorar la adquisición de destrezas y el rendimiento. Entre estas, la práctica mental, según los resultados de este estudio, puede tener un gran impacto.

Este estudio, ha aportado una prueba más de la efectividad del entrenamiento mental, desde la infancia, inclusive; generando mejoras significativas en un tiempo corto, de menos de dos semanas y demostrando la mayor efectividad de una carga de práctica mental con más cantidad de repeticiones de la misma, en el mismo tiempo. Esto abre nuevas perspectivas para la investigación en esta área y para la aplicación sistemática de la práctica mental, al entrenamiento deportivo, desde la niñez.

\section{BIBLIOGRAFÍA}

Betts, G.H. (1909). The distribution and functions of mental imagery. New York Columbia University, Teachers College.

Durand, M., May, C. y Haslam, I.R. (1997).The effects of combining mental and physical practice on motor skill acquisition: A review of literature and some practical implications. The Hong Kong Journal of Sports Medicine and Sports Science, 4,36-41.

Feltz, D.L. y Landers, D.M. (1983). The effects of mental practice on motor skill learning and performance: A metaanalysis. Journal of Sport Psychology, 5, 25-57.

Harris, D.V. y Robinson, W.J. (1986). The effects of skills level on EMG activity during internal and external Imagery. Journal of Sport Psychology, 8, 105-111.

Hird, J.S., Landers, D.M., Thomas, J.R. y Horan, J.J. (1991). Physical practice is superior to mental practice in enhancing cognitive and motor task performance. Journal of Sport and Exercise Psychology, 8, 281-293.

Horn, T.S. (1992). Advances in Sport and Exercise Psychology . Champaigne Illinois. Human Kinetics Publishers.

Jastrow, J.A.(1892). Study of involuntary movements. American Journal of Psychology, 4, 398-407.

Jacobson, E (1932). Electrophysiology of mental activities. American Journal of Psychology, 44, 677-694.

Meacci, W.G. y Price, E.E. (1985). Acquisition and retention of golf putting skill through the relaxation, visualization, and body rehearsal intervention. Research Quarterly for Exercise and Sport, 56, 176-179.

Murphy, S.M. y Jowdy, D.P. (1992). Imagery and Mental Practice. En: T.S. Horn "Advances in Sport and Exercise Psychology" . Champaigne Illinois. Human Kinetics Publishers.

Paivio, A. (1985). Cognitive and motivational functions of imagery in human perfomance. Canadian Journal of Applied Sport Sciences, 10, 22S-28S

Sackett,. R.S. (1934) The Influences of symbolic rehearsal upon the retention of a maze habit. Journal of General Psychology, 10, 376-395.

Perry, H.M. (1939). The relative efficiency of actual and imaginary practice in five selected tasks. Archives of Psychology, 34, 5-75.

Sobalvarro, L. y Salazar, W. (1998) Estado anímico en niñas gimnastas de Centro América y el Caribe previo a la competencia. Estudio no publicado.

Sobalvarro, L. y Salazar, W. (1999) Efecto del tipo de evento competitivo, sobre el estado anímico de niñas gimnastas, pre competencia. Estudio no publicado.

Washburn, M.F. (1916). Movement and mental imagery. Boston: Houghton Mifflin.

Weinberg, R. y Gould, D. (1995). Foundations of Sport and Exercise Psychology. Champaigne, Illinois: Human Kinetics Publishers.

White, A. y Hardy, L. (1998) An in depth analysis of the uses of imagery by high-level canoeists and artistic gymnasts. The Sport Psychologist, 12, 387-403. 